\title{
Rotura esplénica espontánea secundaria a mononucleosis infecciosa
}

\author{
Spontaneous splenic rupture secondary to infectious mononucleosis
}

\author{
Consuelo Gatica', Pablo Soffia², Roberto Charles ${ }^{3}$ y Andrés Vicentela ${ }^{1}$
}

'Programa de Formación en Radiología, Departamento de Imágenes, Facultad de Medicina Clínica Alemana de Santiago-Universidad del Desarrollo. ${ }^{2}$ Departamento de Imágenes, Facultad de Medicina Clínica Alemana-Universidad del Desarrollo.

${ }^{3}$ Departamento de Cirugía, Facultad de Medicina Clínica Alemana-Universidad del Desarrollo.

Declaración de intereses y financiamiento: Los autores declaran no tener conflicto de intereses. No se contó con financiamiento para el desarrollo de este trabajo.

Recibido: 20 de julio de 2020 / Aceptado: 8 de marzo de 2020

\section{Resumen}

La rotura esplénica es una complicación rara pero potencialmente fatal de la mononucleosis infecciosa. Presentamos el caso de una mujer de 18 años que consultó por dolor abdominal de siete días de evolución, asociado a fiebre y pérdida de conciencia brusca y transitoria. En el hemograma presentaba una anemia y linfocitosis. Se realizó una tomografía computada de abdomen y pelvis que mostró un extenso hemoperitoneo, con el bazo rodeado por un hematoma, y numerosas adenopatías cervicales, mesentéricas e inguinales. Se efectuó una laparoscopía que demostró abundante hemoperitoneo con coágulos a lo largo de la gotera parietocólica izquierda. El bazo estaba completamente decapsulado y rodeado por una colección hemática con sangrado en napa. Se realizó una esplenectomía total sin complicaciones. El estudio histopatológico esplénico mostró una atenuación de la pulpa blanca y expansión de la pulpa roja con áreas de hemorragia y necrosis. La IgM anti-cápside para virus de Epstein Barr fue positiva. La paciente evolucionó de manera favorable.

Palabras clave: rotura esplénica; mononucleosis infecciosa; virus Epstein Barr, hemoperitoneo.

\section{Introducción}

L a rotura esplénica espontánea es una complicación infrecuente pero potencialmente fatal de la mononucleosis infecciosa (MI) ${ }^{1}$. La incidencia reportada es de 0,1 a $0,5 \%{ }^{2}$ y ocurre con mayor probabilidad dentro de las primeras cuatro semanas del inicio de los síntomas, afectando a adolescentes y adultos jóvenes de ambos sexos $^{3}$. Si bien el manejo conservador es posible en la mayoría de los pacientes, puede ser necesaria la esplenectomía de urgencia en casos de una hemorragia abdominal intensa ${ }^{4}$. Presentamos el caso de una rotura

\begin{abstract}
Splenic rupture is a rare but potentially fatal complication of infectious mononucleosis. We report the case of an 18-year-old woman, who presented a 7-day history of abdominal pain, sudden temporary loss of consciousness and fever. Admission blood tests showed anemia, and lymphocytosis. Computed tomography of the abdomen and pelvis demonstrated extensive hemoperitoneum and numerous cervical, mesenteric and inguinal enlarged lymph nodes. Laparoscopy was performed and abundant hemoperitoneum with blood clots along the left parietocolic gutter were observed. The spleen was completely decapsulated and surrounded by a hematoma and the subcapsular tissue was bleeding. Total splenectomy was performed without complications. Splenic histology demonstrated white pulp attenuation and expansion of the red pulp with focal hemorrhage and necrosis. IgM anti-viral capsid antigen of Epstein Barr virus was positive. The patient had a satisfactory recovery.

Keywords: splenic rupture; infectious mononucleosis; Epstein Barr virus; hemoperitoneum.
\end{abstract}

esplénica espontánea en una mujer joven cursando con una MI, que se presentó con un abdomen agudo secundario a un hemoperitoneo masivo.

\section{Caso clínico}

Mujer de 18 años, sin antecedentes mórbidos de relevancia, consultó por un dolor abdominal periumbilical e hipogástrico de siete días de evolución, asociado a náuseas y vómitos. Además refería 


\section{Caso Clínico}
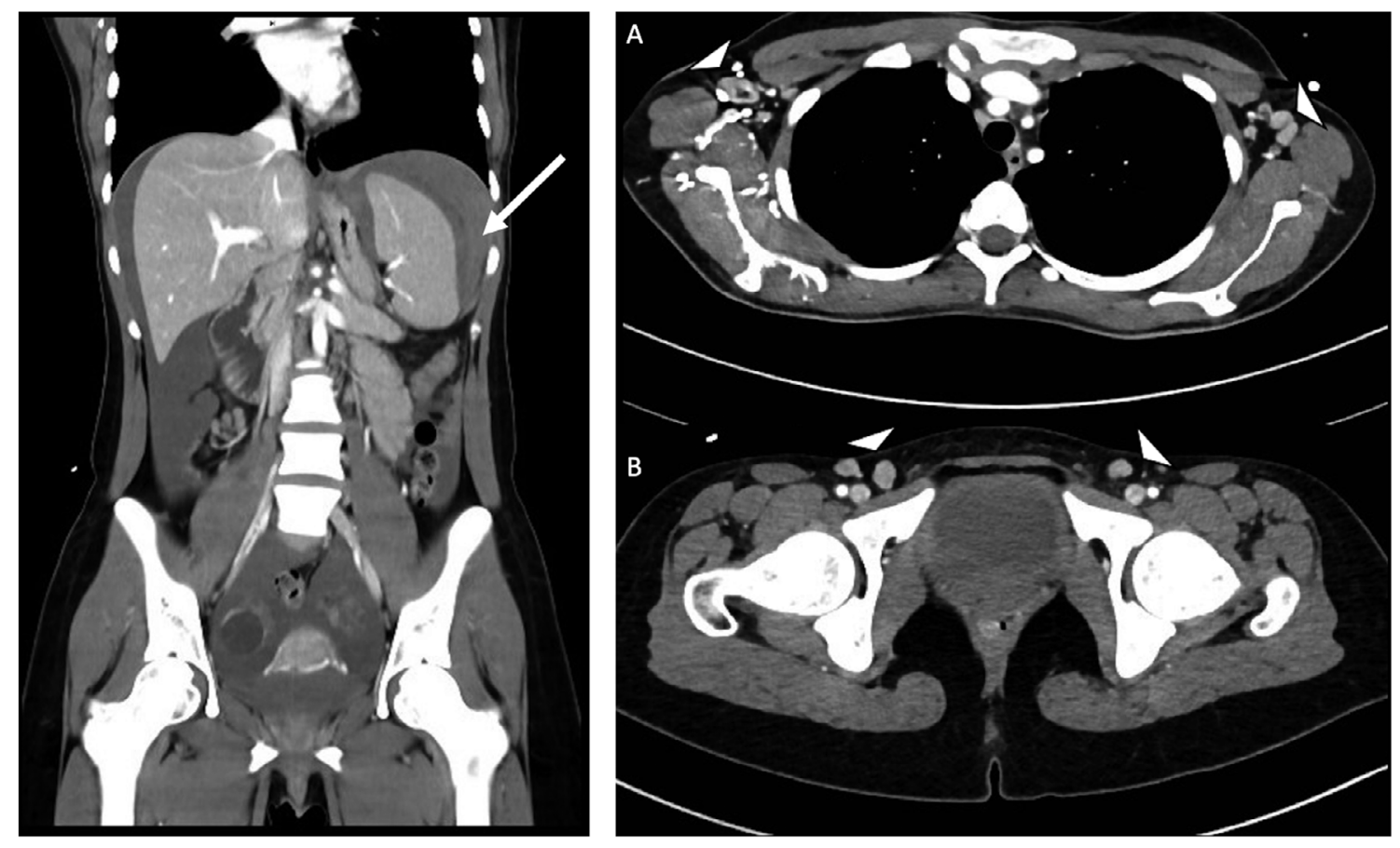

Figura 1. TC de abdomen y pelvis en corte coronal (A) y axial (B). Muestra hemoperitoneo masivo y coágulo periesplénico (flechas blancas).

fiebre hasta $38,5^{\circ} \mathrm{C}$ y un episodio de lipotimia. Ingresó al servicio de urgencia taquicárdica, pálida e hipotensa. Al examen físico presentaba dolor abdominal hipogástrico intenso, sin signos de irritación peritoneal.

Dentro de los exámenes de laboratorio al ingreso destacó una creatininemia de 1,8 mg/dl, transaminasas elevadas: GOT 189 U/1, GGT $202 \mathrm{U} / 1$, fosfatasa alcalina $287 \mathrm{U} / \mathrm{l}$, y un hemograma con hemoglobina $8,9 \mathrm{~g} / \mathrm{dl}$, leucocitos 30.900 céls $/ \mathrm{mm}^{3}$ con $50 \%$ linfocitos, y proteína C-reactiva $6,7 \mathrm{mg} / \mathrm{dl}$.

En la evaluación médica se sospechó un abdomen agudo de origen ginecológico. Se manejó con solución salina y analgésicos i.v. y se realizó una ecografía con protocolo FAST (focused abdominal sonography for trauma) que demostró líquido libre perihepático y en la pelvis. Se solicitó una tomografía computada (TC) de abdomen y pelvis, la que reveló abundante hemoperitoneo y un gran hematoma alrededor del bazo (Figura 1). Además, se identificaron múltiples adenopatías supra e infradiafragmáticas de aspecto inespecífico.

Se realizó una exploración laparoscópica que confirmó un hemoperitoneo de aproximadamente tres litros, con abundantes coágulos en la corredera parietocólica izquierda. Los anexos estaban indemnes, con un cuerpo lúteo derecho sin evidencias de sangrado. Al explorar el hipocondrio izquierdo se observó el bazo completamente decapsulado y rodeado de un gran hematoma asociado a sangrado en napa del tejido esplénico subcapsular. Ante la imposibilidad de preservarlo, se progresó a una laparotomía supraumbilical para una esplenectomía total, la que se efectuó sin complicaciones (Figura 2).

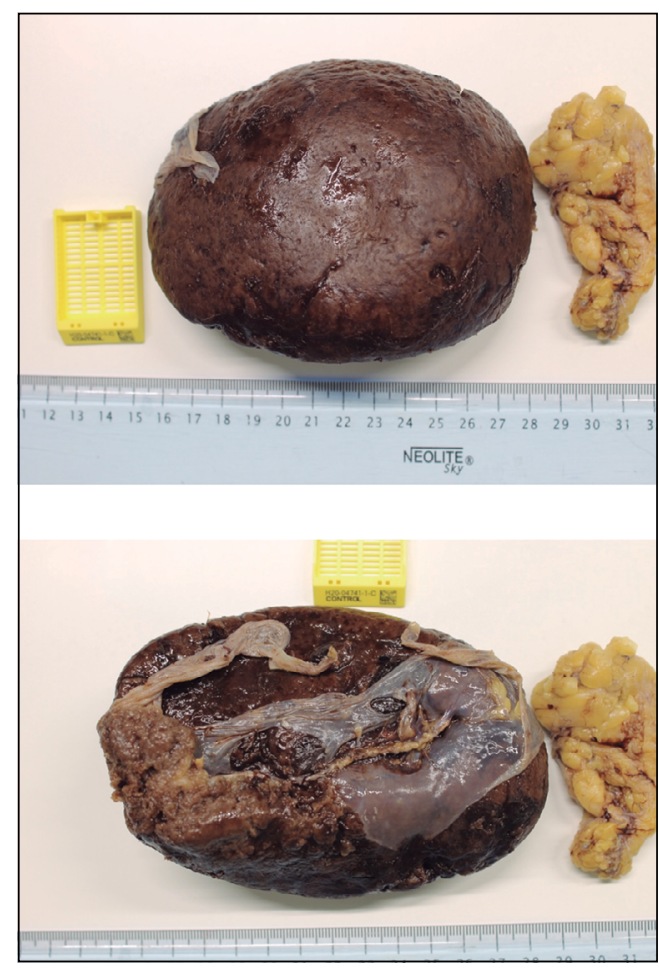

Figura 2. Pieza quirúrgica. Muestra desprendimiento de la cápsula esplénica con serosa opaca y deslustrada. Presenta múltiples focos hemorrágicos en la superficie. 


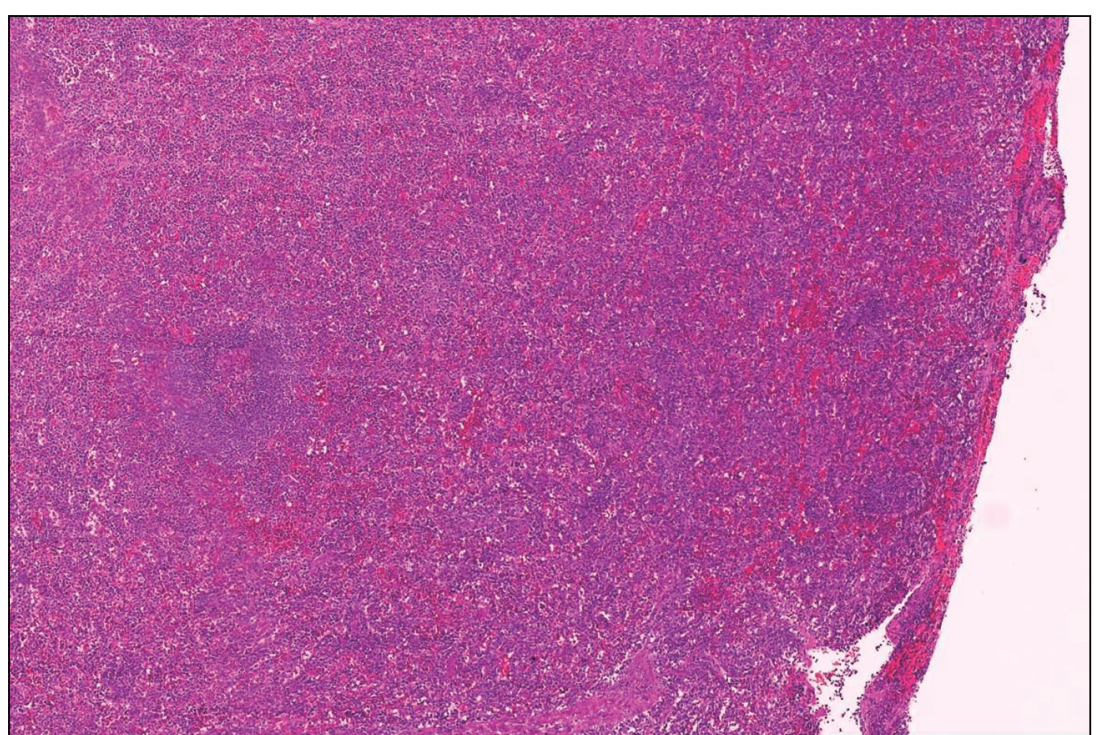

Figura 3. Fotografía microscópica de corte histológico de bazo, aumento 4x. Con tinción HE se observa a este aumento una marcada atenuación de la pulpa blanca y expansión de la pulpa roja, con focos de hemorragia.

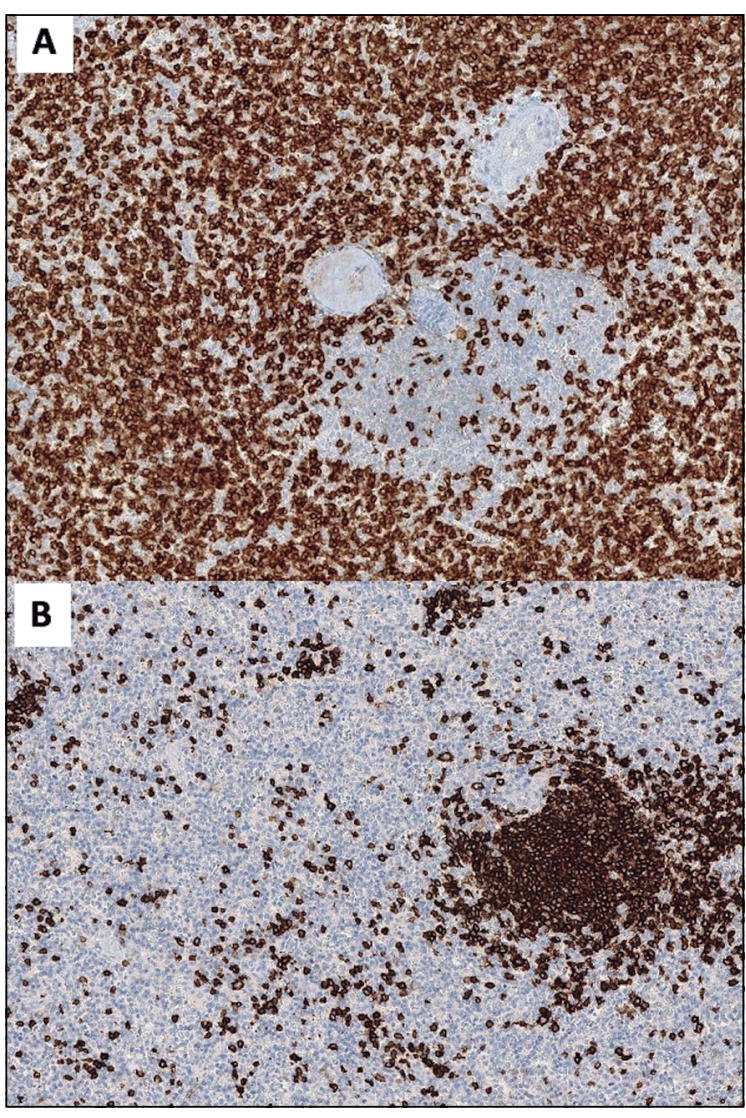

Figura 4. A: Fotografía microscópica del corte histológico de bazo, aumento 10x. El estudio inmunohistoquímico con CD3 muestra una población predominante de linfocitos T, de distribución en pulpa roja. B: Fotografía microscópica de corte histológico de bazo, aumento 10x. El estudio inmunohistoquímico con CD20 muestra menor cuantía de linfocitos $B$, de distribución predominante en la pulpa blanca, con escasos dispersos en la pulpa roja. IgM e IgG anti-cápside para virus Epstein-Barr (VEB), los que resultaron positivos con un índice de 1,78 y 1,84; respectivamente. Adicionalmente se realizó una hibridación in situ cromogénica (CISH) en la muestra de tejido esplénico, siendo positivo para secuencias de VEB. Las otras serologías, IgM para CMV, Brucella spp., virus herpes humano-6 (VH6) y parvovirus fueron negativas. La paciente evolucionó satisfactoriamente y fue dada de alta a las dos semanas.

\section{Discusión}

La MI es una enfermedad linfoproliferativa benigna causada por el VEB. El VEB es un herpesvirus que se transmite por la saliva y tiene un período de incubación de 30 a 50 días. Este agente es altamente prevalente en la comunidad y a los 30 años de edad alrededor de $90 \%$ de la población ha sido expuesta ${ }^{5}$. En la niñez, la infección suele ser asintomática, mientras que en adolescentes y adultos jóvenes se manifiesta clínicamente como una $\mathrm{MI}^{6}$. La mayor parte de los casos ocurren entre los 12 y los 22 años, sin diferencia de género. En los E.U.A, la incidencia es de 45 en 100.000 habitantes 7.

El VEB induce una proliferación de linfocitos $B$ que gatilla una expansión clonal de linfocitos $T$ con hiperplasia linfoide, linfocitosis plasmática y liberación de citoquinas. La presentación clínica suele tener un pródromo de cuatro a cinco días con fiebre y decaimiento, seguido por fiebre, odinofagia, adenomegalia cervical dolorosa, generalmente bilateral y hepato-esplenomegalia. El manejo de esta enfermedad es conservador, con analgesia, antipiréticos e hidratación. Si bien la gran mayoría de los pacientes cursa un cuadro benigno y autolimitado, en un $1 \%$ pueden existir complicaciones graves y eventualmente fatales, entre las que se encuentran la meningoencefalitis, el síndrome de Guillain-Barré, nefritis intersticial aguda, anemia hemolítica, miocarditis, parálisis de pares craneanos, neuropatías, neuritis retrobulbar, trombocitopenia, obs- 
trucción de la vía respiratoria superior y la rotura esplénica espontánea ${ }^{7}$, la que puede ocurrir entre 0,1 y $0,5 \%$ de los casos $^{2}$. Incluso, se ha reportado que hasta $15 \%$ de las roturas esplénicas sin factores de riesgo o enfermedad previamente diagnosticada se deben a una $\mathrm{MI}^{8}$.

El primer caso de rotura esplénica espontánea asociada a una MI fue descrito en 1941. Desde entonces, se han publicado cerca de 100 casos en la literatura médica a nuestro alcance, la mayoría como reportes de casos, principalmente en adolescentes y adultos jóvenes, sin comorbilidades, de los cuales siete resultaron fatales ${ }^{7}$. Una revisión sistemática encontró 85 casos reportados, entre 1985 y 2014 , donde $90 \%$ de los pacientes eran menores de 30 años, con un promedio de edad de 22 años, 70\% varones y la gran mayoría (90\%) no tenía antecedentes mórbidos de relevancia ${ }^{7}$. Mostró además que 14\% de los pacientes relataba un trauma abdominal previo a la rotura, la mayoría de menor intensidad; la mortalidad global fue de $9 \%$. Con respecto al momento de la rotura, ésta varió entre 1 y 54 días desde el inicio de los síntomas y $84 \%$ dentro de las primeras cuatro semanas. Este dato es concordante con una publicación del año 2008 que estudió con ultrasonografía (US) abdominal a 20 deportistas con MI y encontró que el mayor grado de esplenomegalia se registraba como promedio a los 12,3 días después de iniciados los síntomas 9 .

El síntoma más frecuentemente descrito es el dolor abdominal localizado en el hipocondrio izquierdo, presente en $88 \%$ de los pacientes ${ }^{7}$. En los casos fatales reportados, $71 \%$ tuvo además náuseas y vómitos previos al paro cardiorrespiratorio ${ }^{7}$.

Ningún estudio ha logrado determinar signos claros que permitan predecir la probabilidad de una rotura esplénica espontánea en un paciente con MI, aunque sí se ha descrito que hombres menores a 30 años presentan mayor riesgo ${ }^{7}$. Tampoco se ha encontrado una correlación entre la gravedad de los síntomas y el riesgo de rotura esplénica ${ }^{3}$.

Pese a que no hay estudios de calidad que determinen el riesgo del ejercicio en pacientes con MI, la recomendación general es evitar la actividad física intensa por cuatro semanas desde el inicio de los síntomas ${ }^{3}$. En pacientes deportistas se recomienda esperar la resolución de la sintomatología y realizar una evaluación ecográfica para estimar el tamaño del bazo, sobretodo en aquellos que practican deportes de contacto ${ }^{7}$.

En la actualidad, los pacientes con dolor abdominal agudo que están cursando una MI suelen ser sometidos a exploración por métodos de diagnóstico por imágenes, dada su alta disponibilidad, sensibilidad y especificidad para detectar causas quirúrgicas. Los métodos más utilizados son la US y la TC de abdomen y pelvis. En el caso de la rotura esplénica, los hallazgos en la US incluyen esplenomegalia, áreas hipoecogénicas en el parénquima esplénico, signos de fractura del bazo y hemoperitone $o^{10}$. También se puede identificar líquido subcapsular, aunque inicialmente el coágulo periesplénico puede ser indistinguible del resto del bazo, dado que su ecogenicidad puede ser muy similar al tejido adiposo intraabdominal $^{11}$. La US con estudio Doppler permite además evaluar la vascularización del bazo, que puede ser importante al momento de decidir la conducta terapéutica. En la TC, los signos típicos de rotura esplénica son laceraciones, fracturas o estallido del parénquima y hemoperitoneo de cuantía variable ${ }^{12}$. En el caso de las roturas no traumáticas, el signo más frecuente es el hematoma periesplénico, que puede tener distinta densidad dependiendo de la antiguedad del coágulo ${ }^{13}$. En etapas agudas, el hematoma muestra una densidad elevada y es conocido como el signo del "coágulo centinela" que orienta al origen del hemoperitoneo, incluso cuando no existen alteraciones visibles en el órgano adyacente ${ }^{14}$.

Existen numerosos reportes de casos que muestran el éxito del manejo conservador de la rotura esplénica, con estrecha observación clínica y seguimiento con imágenes, principalmente US y $\mathrm{TC}^{7}$. En este sentido, si bien la exposición a radiación ionizante puede ser un factor de preocupación en la población joven, el riesgo relativo de la esplenectomía es considerado mayor, por lo que el balance de beneficio versus riesgo es a favor del uso de imágenes radiantes como seguimiento.

Dentro de las complicaciones infecciosas de la esplenectomía destaca un riesgo elevado de sufrir infecciones graves por bacterias encapsuladas (Streptococcus pneumoniae, Haemophilus influenzae y Neisseria meningitidis), parásitos y otros agentes, en las que el bazo desempeña un papel importante en su eliminación a través de las funciones de filtración, remoción, fagocitosis y producción de opsoninas ${ }^{15}$. Los riesgos de sepsis y mortalidad relacionada con la sepsis parecen ser aproximadamente de dos a tres veces mayores en los pacientes asplénicos en comparación con la población genera ${ }^{16}$. En un estudio de cohorte que evaluó a más de 8.000 pacientes esplenectomizados durante un período de 27 años, se identificaron mayores tasas de sepsis y mortalidad relacionada con la sepsis en comparación con los controles. El mayor riesgo de sepsis y mortalidad se mantuvo durante un período de 10 años y se presume que es de por vida ${ }^{17}$.

Durante la última década se han publicado experiencias del manejo de la rotura esplénica con técnicas de radiología intervencional, principalmente usando embolización con partículas o dispositivos metálicos ${ }^{18}$.

La mortalidad de la rotura esplénica no traumática es de $9 \%$, lo que la sitúa levemente por debajo de la mortalidad por la de origen traumática que es del 14\%. Otras causas poco frecuentes de rotura esplénica no traumática incluyen otras etiologías infecciosas (malaria, fiebre glandular), hematológicas (linfoma) o sistémicas (sarcoidosis) ${ }^{19}$. 


\section{GrCi cuso cinito}

\section{Conclusión}

La rotura esplénica espontánea es una complicación infrecuente pero potencialmente fatal de la mononucleosis infecciosa que en la mayoría de los casos requerirá sólo de tratamiento conservador. Dada la alta prevalencia de la infección en la población general, es relevante conocer sus manifestaciones y forma de estudio para un diagnóstico oportuno, así como un manejo correcto y exitoso de esta complicación.

\section{Referencias bibliográficas}

1.- Dunmire S K, Hogquist K A, Balfour H H. Infectious mononucleosis. Curr Top Microbiol Immunol 2015; 390(Pt 1): 211-40. doi: 10.1007/978-3-319-22822-8_9.

2.- Greydanus D E, Gregoire Bottex M M. The dark side of MONO. Acad J Ped Neonatol. 2017; 5(3): 555718. doi: 10.19080/ AJPN.2017.05.555718.

3.- Foreman B H, Mackler L, Drew Malloy E. Clinical inquiries. Can we prevent splenic rupture for patients with infectious mononucleosis? J Fam Pract 2005; 54: 547-8.

4.- Won A C M, Ethell A. Spontaneous splenic rupture resulted from infectious mononucleosis. Int J Surg Case Rep 2012; 3: 97-9. doi: 10.1016/j.ijscr.2011.08.012.

5.- Kinderknecht $J$ J. Infectious mononucleosis and the spleen. Curr Sports Med Rep 2002; 1:11620. doi: 10.1249/00149619-200204000-00009.

6.- Frank E A, LaFleur J R, Okosun S. Nontraumatic splenic rupture due to infectious mononucleosis. J Acute Care Surg 2019; 9: 69 71. doi: 10.17479/jacs.2019.9.2.69.

7.- Bartlett A, Williams R, Hilton M. Splenic rupture in infectious mononucleosis: A systematic review of published case reports. Injury 2016; 47: 531-8. doi: 10.1016/j. injury.2015.10.071.

8.- Siliézar M M, Muñoz C C, Solano-Iturri J D, Ortega-Comunian L, Mollejo M, MontesMoreno S, et al. Spontaneously ruptured spleen samples in patients with infectious mononucleosis. Analysis of histology and lymphoid subpopulations. Am J Clin Pathol 2018; 150: 310-7. doi: 10.1093/ajcp/aqy056.

9.- Hosey R G, Kriss V, Uhl T L, DiFiori J, Hecht S, Wen D Y. Ultrasonographic evaluation of splenic enlargement in athletes with acute infectious mononu- cleosis. $\mathrm{Br} \mathrm{J}$ Sports Med 2008; 42: 974-7. doi: 10.1136/ bjsm.2008.050807.

10.- Johnson M A, Cooperberg P L, Boisvert J, Stoller J L, Winrob H. Spontaneous splenic rupture in infectious mononucleosis: sonographic diagnosis and follow-up. AJR 1981; 136: 111-4. doi: 10.2214/ajr.136.1.111.

11.- Wilson R L, Rogers W F, Shaub M S, Binnbaum W. Splenic subcapsular hematoma-ultrasonic diagnosis. West J Med 1978; 128: 6-8.

12.- Hassan R, Abd Aziz A, Md Ralib AR, Saat A. Computed tomography of blunt spleen injury: a pictorial review. Malays J Med Sci. 2011;18: 60-7.

13.- Weaver H, Kumar V, Spencer K, Maatouk M, Malik S. Spontaneous splenic rupture: A rare life-threatening condition; diagnosed early and managed successfully. Am J Case Rep 2013; 14:13-5. doi: 10.12659/AJCR.883739.

14.- Orwig D, Federle M P. Localized clotted blood as evidence of visceral trauma on CT: the sentinel clot sign. AJR Am J Roentgenol 1989; 153: 747-9. doi: 10.2214/ajr.153.4.747.

15.- Cofré F, Cofré J. Asplenia e hiposplenia en pediatría. Prevención de sepsis bacteriana fuminante. Rev Chilena Infectol 2014; 31: 6672. doi: 10.4067/S0716-10182014000100010.

16.- Thomsen R W, Schoonen W M, Farkas D K, Riis A, Jacobsen J, Fryzek J P, et al. Risk for hospital contact with infection in patients with splenectomy: a population-based cohort study. Ann Intern Med 2009; 151: 546-55. doi: 10.7326/0003-4819-151-8-20091020000008 .

17.- Kristinsson S Y, Gridley G, Hoover R N, Check D, Landgren O. Long-term risks after splenectomy among 8,149 cancer-free American veterans: a cohort study with up to 27 years follow-up. Haematologica 2014; 99: 392-8. doi: 10.3324/haematol.2013.092460.

18.- Ahuja C, Farsad K, Chadha M. An overview of splenic embolization. AJR Am J Roentgenol 2015; 205: 720-5. doi: 10.2214/AJR.15.14637.

19.- Knoblich R. Pathologic (so-called spontaneous) rupture of spleen in leukemia and lymphoma. Mich Med 1996; 65: 105-10. 